\section{Malignant rhabdoid tumor of the liver: a case report and literature review}

\author{
Satoru Oita, ${ }^{1}$ Keita Terui, 1 \\ Syugo Komatsu,2 Tomoro Hishiki, 2 \\ Takeshi Saito,1 Tetsuya Mitsunaga,1 \\ Mitsuyuki Nakata, ${ }^{1}$ Hideo Yoshida ${ }^{1}$ \\ 1Department of Pediatric Surgery, \\ Graduate School of Medicine, Chiba \\ University; 2Department of Pediatric \\ Surgery, Chiba Children's Hospital, Japan
}

\section{Abstract}

Malignant rhabdoid tumor (MRT) is a rare and aggressive malignancy associated with poor outcomes. MRT of the liver is even rarer, and little information has been described. We report the case of an 8-month-old boy with MRT of the liver. The tumor showed aggressive progression despite a multidisciplinary approach, and the patient died due to multiple organ failure 14 days after admission. Autopsy revealed the liver tumor and multiple metastases with negative immunohistochemistry for INI1/BAF47. A review of 53 cases of pediatric MRT of the liver is provided.

\section{Introduction}

Malignant rhabdoid tumor (MRT) is a rare and aggressive malignancy associated with poor outcomes. MRT of the liver is even rarer, and little information is shared.1,2 We report a case of MRT of the liver and provide a literature review of MRT of the liver in children.

\section{Case Report}

An 8-month-old boy was referred with a 5day history of fever, cough, and loss of appetite. He had been well until then, and had no past history. On admission, he was $70.5 \mathrm{~cm}$ tall [0.0 standard deviations (SD)] and weighted $7.3 \mathrm{~kg}$ (+1.0 SD). Body temperature was $38.3^{\circ} \mathrm{C}$, blood pressure was $96 / 54 \mathrm{mmHg}$, heart rate was 140 beats/min and respiratory rate was 30 breaths/min. His level of consciousness was good. Physical examination showed abdominal distension and hepatomegaly. Laboratory tests showed elevated levels of white blood cells $(17,600 / \mu \mathrm{L})$, C-reactive protein $(4.4 \mathrm{mg} / \mathrm{dL})$, lactate dehydrogenase (3131 U/L) and liver enzymes. Levels of $\alpha$-fetoprotein (AFP, 490.3 $\mathrm{ng} / \mathrm{mL}$ ) and neuron-specific enolase (NSE, 561 $\mathrm{ng} / \mathrm{mL}$ ) were elevated. Urine levels of vanillyl mandelic acid and homovanillic mandelic acid were normal. Computed tomography revealed multiple low-attenuation tumors throughout the liver (Figure 1). Both meta-iodobenzylguanidine (MIBG) scintigraphy and bone scan yielded negative results. The provisional diagnosis was neuroblastoma with metastasis (Stage 4S), although negative results were obtained for MIBG scintigraphy.

During these examinations, abdominal distension rapidly worsened. Nine days after admission, open biopsy was performed uneventfully. The day after surgery, chemotherapy including vincristine $(0.05$ $\mathrm{mg} / \mathrm{kg} / \mathrm{day}$ ) and cyclophosphamide (30 mg/kg) was started. On postoperative day 2, continuous hemodiafiltration was applied to manage tumor lysis syndrome and anuria. On postoperative days 3 and 4, radiotherapy of 1.5 Gy/day was performed. Despite these treatments, the patient died 14 days after admission due to multiple organ failure.

On autopsy, the liver was enlarged and weighed $1224 \mathrm{~g}$ (Figure 1). The largest of the tumors, $5 \mathrm{~cm}$ in diameter, was found in the posterior right lobe of the liver. Tumor cells had infiltrated invasively throughout the liver and half of the tumor was necrotic. Metastases were found in the right adrenal gland, bilateral lungs, and regional lymph nodes. The tumor cells showed eccentric vesicular nuclei, large nucleoli, abundant eosinophilic cytoplasm and periodic acid-Schiff reagent-positive cytoplasmic inclusion. Immunohistochemistry showed positive results for vimentin, epithelial membrane antigen, and cytokeratin 19, with focally positive results for cytokeratin 7 and NSE, and negative results for S-100, AFP and INI1/BAF47. Histological diagnosis was MRT of the liver.

\section{Literature review}

Starting from a previous review of 34 cases, 1 we collected 53 pediatric cases of MRT of the liver by adding the present case, 8 cases from the English and French literature, and 10 cases from the Japanese literature..$^{3-9}$ Median patient age was 8.0 months (range, 0 days to 15 years). Three cases (5.7\%) were neonates, 35 (66.0\%) were less than 1 year old, and 47 (88.7\%) were less than 3 years old. Males comprised $56.0 \%$ of patients. Almost all presenting symptoms were nonspecific, such as abdominal mass in $35.8 \%$, fever in $26.4 \%$, oral feeding difficulty or vomiting in $20.8 \%$, abdominal distention in $17.0 \%$ and abdominal pain in $7.5 \%$.

Alpha-fetoprotein is one of the most important pieces of information allowing differentiation between hepatoblastoma and other neoplasms of the liver. AFP levels were reported in
Correspondence: Keita Terui, Department of Pediatric Surgery, Graduate School of Medicine, Chiba University, 1-8-1, Inohana, Chuou-ku, Chiba 260-8677, Japan.

Tel.: +81.43.222.7171 - Fax: +81.43.226.2366

E-mail: kta@cc.rim.or.jp

Key words: liver tumor, rhabdoid tumor, abdominal neoplasms, neoplasm metastasis, SWI-SNF-B chromatin-remodeling complex.

Contributions: SO wrote the manuscript under supervision of KT; SK performed the statistical analysis and the review of the literature; TS, TM and MN provided medical care, including the surgical procedure; HY designed the study and reviewed the manuscript.

Conflict of interests: the authors declare no potential conflict of interests.

Received for publication: 30 July 2014.

Revision received: 6 January 2015.

Accepted for publication: 6 January 2015.

This work is licensed under a Creative Commons Attribution NonCommercial 3.0 License (CC BYNC 3.0).

(C) Copyright S. Oita et al., 2015

Licensee PAGEPress, Italy

Pediatric Reports 2015; 7:5578

doi:10.4081/pr.2015.5578

15 cases, with 4 cases (26.7\%) showing relatively high levels of AFP, according to agematched standard values for AFP.10 The highest AFP level was only $13,381 \mathrm{ng} / \mathrm{mL}$ at 90 days old, 11 meaning that the involvement of hepatoblastoma could be ruled out. The most specific finding for MRT is thought to be immunohistochemical suppression of INI1/BAF47.12,13 Immunohistochemical evaluation of INI1/BAF47 was performed in 20 cases (37.7\%), all of which showed no nuclear expression. INI1/BAF47 evaluation has obviously gained importance, and was actually used in $72 \%$ of cases in the last decade (20052014).

Metastatic disease was present in $76.1 \%$ of patients (35/46). The most common sites of metastasis were the lungs (65.6\%) and lymph nodes (34.4\%), followed by the central nervous system $(9.4 \%)$, skin $(6.3 \%)$, bone $(6.3 \%)$, and other tissues (15.6\%), including the adrenal glands, peritoneum, retroperitoneum, mesentery, and pleura. Of the 35 cases with metastasis, only 1 patient survived; surprisingly, that patient survived despite incomplete resection of the primary tumor. ${ }^{14}$ Of the 11 cases without metastasis, 3 survived after complete surgical resection. Only 5 patients (9.4\%) remained alive at the time of reporting or on follow-up (data included information obtained by per- 
sonal communication). To characterize these 5 survivors, we compared them with fatal cases in the following 6 factors: sex; age ( $>1$ year old); metastasis; complete resection; chemotherapy; and radiation (Table 1). The proportions of survivor and non-survivor cases $>1$ year old were $80.0 \%$ and $23.4 \%$, respectively; the rates of no metastasis were $75 \%$ and $19 \%$, respectively; the rates of complete resection were $75.0 \%$ and $31.1 \%$, respectively. Among patients who died, median duration of survival after first medical contact was 1.5 months (range, 2 days to 18 months), with 19 patients (47.5\%) dying within 1 month.

Various treatment regimens of chemotherapy were reported, and a wide variety of agents were used. The most commonly used were doxorubicin (21 patients), vincristine (21 patients), etoposide (19 patients), cisplatin (19 patients), carboplatin (17 patients), ifosfamide (14 patients) and cyclophosphamide (14 patients). The agents most commonly used by survivors were vincristine (4 of 4 survivors), ifosfamide (3 of 4 survivors), cyclophosphamide (3 of 4 survivors), and doxorubicin (3 of 4 survivors). The noteworthy patient who survived despite the presence of residual tumor had received chemotherapy including 5 cycles of vincristine/adriamycin/ cyclophosphamide followed by ifosfamide/etoposide for 7 months. ${ }^{14}$

To evaluate the effects of each agent on duration of survival, Kaplan-Meier survival analysis was performed. Non-surviving cases with specific descriptions of both content of chemotherapy and duration of survival from first medical contact were included in this analysis. Among the seven most commonly used agents (doxorubicin, vincristine, etoposide, cisplatin, carboplatin, ifosfamide and cyclophosphamide), only frequency of ifosfamide use differed significantly between the administered group and the non-administered group ( $\mathrm{P}=0.046$ ) (Figure 2). Age of onset in the administered and non-administered groups was 6.0 and 1.0 months, respectively $(\mathrm{P}=0.027$, Mann-Whitney $\mathrm{U}$ test). Various agents, including etoposide (7/9), carboplatin (6/9), vincristine (5/9), cisplatin (4/9), cyclophosphamide (3/9), actinomycin D (3/9), and doxorubicin (2/9), were combined with ifosfamide. Radiotherapy was performed for 3 patients, but all died.

\section{Discussion}

Malignant rhabdoid tumor was originally described as a highly malignant variant of Wilms' tumor in 1978,15 and was designated as a distinct entity in 1982.16 Outcomes for this rare and aggressive malignancy are usually poor. MRT of the liver is even rarer, and little information has been accumulated. According to a database of 157 cases of MRT in children and adolescents, ${ }^{17}$ the following three main areas of occurrence are recognized: the central nervous system (50\%); kidneys (25\%); and soft tissue (19\%). MRT of the liver was reported for only 1 case $(0.6 \%)$ in that database.

The present case was thought to be fairly typical of MRT of the liver, showing infantile onset, non-specific symptoms, resistance to chemotherapies, and rapid death following aggressive progression. Preoperative diagnosis was difficult, largely because the rarity of MRT involving the liver means that characteristic imaging findings have not been reported. AFP level was not particularly high (490.3 $\mathrm{ng} / \mathrm{mL}$ ), which ruled out hepatoblastoma. However, misdiagnosis resulted from the diffuse metastases in the liver resembling the findings in neuroblastoma Stage $4 \mathrm{~S}$, even though MIBG scintigraphy yielded negative results. Definitive diagnosis was obtained by

Table 1. Comparison between survivors and non-survivors of malignant rhabdoid tumor.

\begin{tabular}{lcc} 
& Survivors $(\mathrm{n}=5)$ & Non-survivors $(\mathbf{n}=4 \mathbf{7})$ \\
Male & $1 / 5(20.0 \%)$ & $26 / 43(60.5 \%)$ \\
$>$ l year old & $4 / 5(80.0 \%)$ & $11 / 47(23.4 \%)$ \\
\hline No metastasis & $3 / 4(75.0 \%)$ & $8 / 42(19.0 \%)$ \\
Complete resection & $3 / 4(75.0 \%)$ & $14 / 45(31.1 \%)$ \\
\hline Chemotherapy & $5 / 5(100 \%)$ & $39 / 45(86.7 \%)$ \\
Radiation & $0 / 4(0 \%)$ & $3 / 45(6.7 \%)$ \\
\hline
\end{tabular}
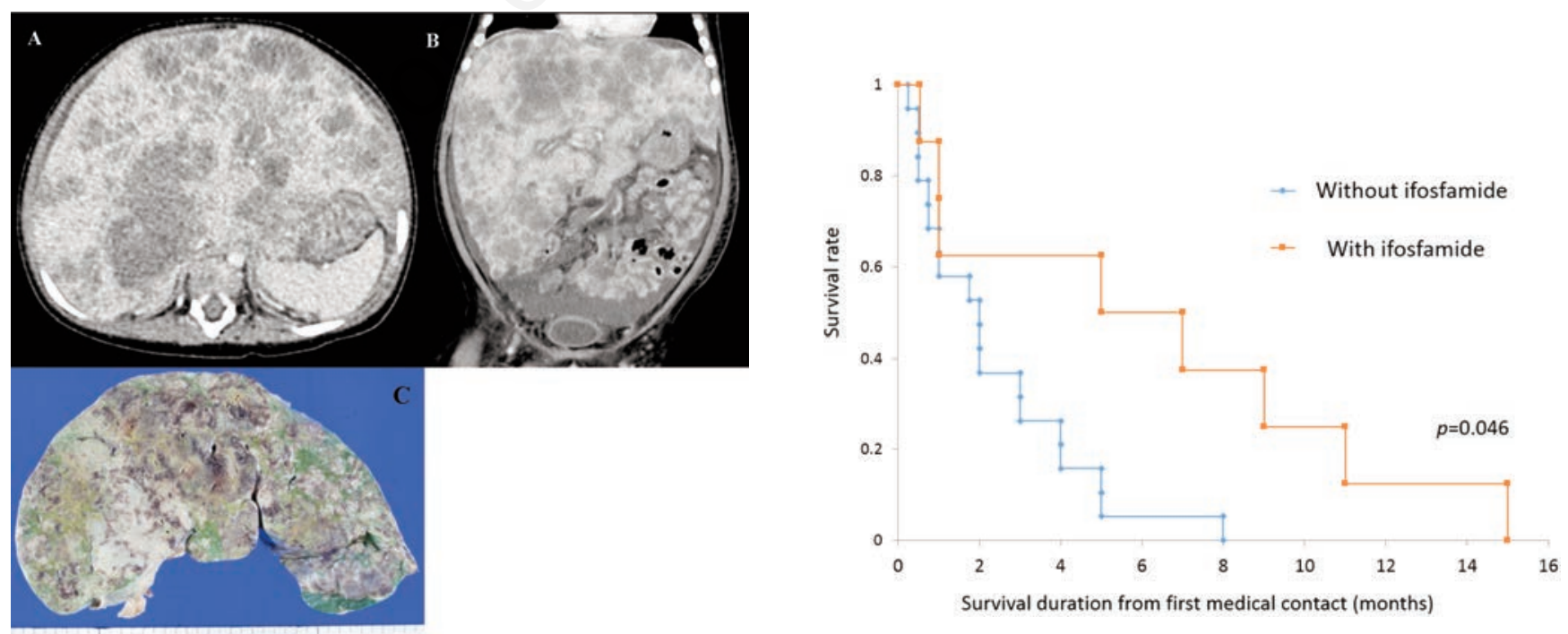

Figure 1. Axial (A) and coronal-view (B) computed tomography, and autopsy specimen (C) of the liver. Multiple tumors are seen throughout the enlarged liver.

Figure 2. Survival curves for non-survivors treated by chemotherapy with/without ifosfamide. 
immunohistochemical evaluation of INI1/BAF47 in the biopsy specimen.

Starting with the previous review of 34 cases, ${ }^{1}$ we collected a total of 53 pediatric cases of MRT of the liver by adding 19 cases. Age, sex, presenting symptoms, and outcomes for the 53 cases remained about the same as those reported previously. ${ }^{1}$ Survivors tended to be older, were more likely to be female, tended to have no metastases, and received complete resection. These tendencies were also described in the previous review and in research using the SEER (Surveillance, Epidemiology, and End Results) database that included 229 cases of MRT of various origins. ${ }^{1,5}$ The only new knowledge provided in the present review appears to be small clues about chemotherapeutic options; among the 7 most commonly used agents (doxorubicin, vincristine, etoposide, cisplatin, carboplatin, ifosfamide and cyclophosphamide), only ifosfamide was associated with significant extension of survival in non-surviving cases. Based on experience with 13 pediatric cases of extracranial MRT of various origins, Gururangan et al. also reported the efficacy of ifosfamide; 18 however, these analyses showed considerable biases. In particular, factors influencing outcomes (e.g., age of onset) were discounted. Furthermore, the number of cases was too small, and no reasons have been suggested as to why ifosfamide might have a specific effect. Further studies are needed to clarify the optimal therapeutic approaches. ${ }^{19}$

\section{Conclusions}

We have presented a case of MRT of the liver with aggressive progression despite a multidisciplinary approach. A review of the literature showed that MRT of the liver occurs mainly in infants under 3 years old, with non-specific symptoms and a high frequency of metastasis. Tumor progression is so aggressive that survival remains dismal if treatment proves unsuccessful. Risk factors associated with poor prognosis were age $<1$ year old and presence of metastasis, and definitive therapeutic options to improve outcomes remain unclear. Aggressive chemotherapy with complete resection may represent a means to rescue patients. Alkylators might be key drugs for MRT in the liver, and further studies are needed to determine optimal combinations of agents.

\section{References}

1. Trobaugh-Lotrario AD, Finegold MJ, Feusner JH. Rhabdoid tumors of the liver: rare, aggressive, and poorly responsive to standard cytotoxic chemotherapy. Pediatr Blood Cancer 2011;57:423-8.

2. Kerl K, Holsten T, Frühwald MC. Rhabdoid tumors: clinical approaches and molecular targets for innovative therapy. Pediatr Hematol Oncol 2013;30:587-604.

3. Beckwith JB, Palmer NF. Histopathology and prognosis of Wilms tumors: results from the First National Wilms' Tumor Study. Cancer 1978;41:1937-48.

4. Gonzalez-Crussi F, Goldschmidt RA, Hsueh $\mathrm{W}$, et al. Infantile sarcoma with intracytoplasmic fi lamentous inclusions: distinctive tumor of possible histiocytic origin. Cancer 1982;49:2365-75.

5. Sultan I, Qaddoumi I, Rodríguez-Galindo $\mathrm{C}$, et al. Age, stage, and radiotherapy, but not primary tumor site, affects the outcome of patients with malignant rhabdoid tumors. Pediatr Blood Cancer 2010;54:3540.

6. White FV, Dehner LP, Belchis DA, et al. Congenital disseminated malignant rhabdoid tumor: a distinct clinicopathologic entity demonstrating abnormalities of chromosome 22q11. Am J Surg Pathol 1999;23:249-56.

7. Clairotte A, Ringenbach F, Laithier V, et al. Malignant rhabdoid tumor of the liver with spontaneous rupture: a case report. Ann Pathol 2006;26:122-5.

8. Bourdeaut F, Fréneaux $\mathrm{P}$, Thuille B, et al. Extra-renal non-cerebral rhabdoid tumours. Pediatr Blood Cancer 2008;51:363-8.

9. Abe T, Oguma E, Nozawa $K$, et al.
Malignant rhabdoid tumor of the liver: a case report with US and CT manifestation. Jpn J Radiol 2009;27:462-5

10. Machado I, Noguera R, Santonja N, et al. Immunohistochemical study as a tool in differential diagnosis of pediatric malignant rhabdoid tumor. Appl Immunohistochem Mol Morphol 2010;18: 150-8.

11. Martelli MG, Liu C. Malignant rhabdoid tumour of the liver in a seven-month-old female infant: a case report and literature review. Afr J Paediatr Surg 2013;10:50-4.

12. Agarwala S, Jindal B, Jana M, et al. Malignant rhabdoid tumor of liver. J Indian Assoc Pediatr Surg 2014;19:38-40.

13. Blohm ME, Vesterling-Hörner D, Calaminus G, et al. Alpha 1-fetoprotein (AFP) reference values in infants up to 2 years of age. Pediatr Hematol Oncol 1998;15:135-42.

14. Wagner LM, Garrett JK, Ballard ET, et al. Malignant rhabdoid tumor mimicking hepatoblastoma: a case report and literature review. Pediatr Dev Pathol 2007;10: 409-15.

15. Al Nassan A, Sughayer M, Matalka I, et al. INI1 (BAF 47) immunohistochemistry is an essential diagnostic tool for children with hepatic tumors and low alpha fetoprotein. J Pediatr Hematol Oncol 2010;32:e7981.

16. Wilson BG, Roberts CW. SWISNF nucleosome remodellers and cancer. Nat Rev Cancer 2011:11:481-92.

17. Wu X, Dagar V, Algar E, et al. Rhabdoid tumour: a malignancy of early childhood with variable primary site, histology and clinical behaviour. Pathology 2008;40:66470.

18. Gururangan S, Bowman LC, ParhamDM, et al. Primary extracranial rhabdoid tumors. Clinicopathologic features and response to ifosfamide. Cancer 1993;71:2653-9.

19. Rosson GB, Vincent TS, Oswald BW, et al. Drug resistance in malignant rhabdoid tumor cell lines. Cancer Chemother Pharmacol 2002;49:142-8. 